\title{
Oligodendroglioma: an analysis of 42 cases
}

\author{
IMS WILKINSON,* JR ANDERSON, † AE HOLMES* \\ From the Departments of Neurological Surgery and Neurology* and Histopathology, $\dagger$ Addenbrooke's Hospital, \\ Cambridge, UK
}

SUMMARY This paper presents the clinical and pathological data obtained from 42 patients with oligodendroglioma diagnosed over a 20 year period at Addenbrooke's Hospital, Cambridge. In all cases the diagnosis was established unequivocally by histological examination. The principal conclusions of this study were: (1) the outcome was not as favourable as anticipated, nine patients dying within 1 year of diagnosis, and only 10 patients surviving 5 years, (2) age at the time of diagnosis was the single most important prognostic factor, patients under 45 years at diagnosis having a significantly greater survival time than older patients, (3) two other factors had a significantly adverse effect on prognosis, namely the presence of a focal neurological deficit at the time of diagnosis and the presence of nuclear pleomorphism in the tumour when examined histologically, (4) although cells containing glial fibrillary acidic protein were demonstrated in almost every tumour, their number and distribution did not appear to influence the prognosis.

Oligodendroglioma is less common than astrocytoma and generally considered to have a better prognosis, but it is also described as unpredictable with poor correlation between histological evidence of malignancy and survival. ${ }^{1-4}$ Its degree of prognostic uncertainty merits reassessment of its properties. Hence, the objectives of this study were two-fold, firstly to re-examine the clinical features of oligodendroglioma, and secondly to determine if any factors, clinical, pathological or therapeutic, had a significant influence on survival. The recently developed immunoperoxidase technique for the demonstration of glial fibrillary acidic protein (GFAP) was included in the search for predictive histological features.

\section{Patients and methods}

\section{Clinical}

Forty-two patients with pure oligodendroglioma were diagnosed and treated at Addenbrooke's Hospital, Cambridge, between 1961 and 1984 . The hospital records of these patients enabled an accurate review of the clinical features at the time of diagnosis, of the operative findings and of the surgical and radiotherapeutic schedules.

The initial presenting symptom was recorded as epilepsy,

Address for reprint requests: Dr IMS Wilkinson, Department of Neurological Surgery and Neurology, Addenbrooke's Hospital, Cambridge CB2 2QQ, UK.

Received 29 January 1986 and in revised form 19 April 1986. Accepted 25 April 1986 slowly evolving neurological deficit, raised intracranial pressure or stroke. Morbidity by the time of diagnosis was documented separately. Radiological data provided evidence of tumour calcification. The surgical data provided the precise tumour location and the nature of the surgical procedure, either needle biopsy, subtotal removal or macroscopic removal. The timing and amount of post-operative irradiation was recorded. Finally, as almost all patients were followed up regularly at Addenbrooke's Hospital or within the East Anglian region their length of survival and status in July 1984 were known. Accurate follow-up has been possible in 41 of the 42 patients, one having been lost to follow-up 4 years after operation.

\section{Histology}

Patient selection was based on the original histology report. All the neurosurgical biopsies had been examined initially by a neuropathologist and only 42 patients with a definite report of oligodendroglioma were included in this study. Where the histological diagnosis was in doubt or when astrocytoma co-existed with oligodendroglioma, the patient was excluded from the survey. In every case histological assessment was based on surgical material. Specimen size varied but all the tissue obtained by biopsy had been fixed in buffered formal saline, paraffin embedded and sectioned for histology.

In 1984-85 the histology of the 42 tumours was reviewed by one of us and on the basis of a haematoxylin and eosin stain all were considered acceptable as oligodendroglioma. Several morphological features were employed in a grading system as detailed in table 1. The histological criteria of malignancy were similar to those employed in the Kernohan system of grading astrocytomas where it has been shown to be of prognostic significance. ${ }^{5-9}$ It was considered practical to divide the oligodendrogliomas into only three grades. 
Table 1 Oligodendroglioma-grading system

\begin{tabular}{lll}
\hline $\begin{array}{l}\text { Cell density } \\
\text { Mitoses per 10 high power fields }-1 / 2 / 3\end{array}$ & Grade $1-3-4$ \\
(0-2, 5-9, 10+) & & \\
$\begin{array}{l}\text { Nuclear pleomorphism } \\
\text { Vascular endothelial }\end{array}$ & $-1 / 2 / 3$ & Grade 2-5-6 \\
$\begin{array}{l}\text { cell proliferation } \\
\text { Necrosis }\end{array}$ & $-0 / 1$ & Grade 3-7-10 \\
\end{tabular}

Histological parameters considered to be indicative of malignancy were scored on a simple point system and the total scores were assigned to 3 grades.

Thus grade 1, expected to be the most benign, showed low cell density, uniform nuclei, less than 2 mitoses per 10 high power field and lacked areas of necrosis or vascular endothelial proliferation. Grade 3, considered the most malignant, showed high cell density, marked nuclear pleomorphism, numerous mitoses and often included necrotic foci and vascular endothelial cell proliferation. Tumours between these two extremes were placed in the intermediate grade 2 category. (Cell density was defined on the basis of proximity of tumour cell nuclei, as seen in $5 \mu \mathrm{m}$ sections stained with haematoxylin and eosin. In tumours of low cell density the nuclei were at least their own width apart. In tumours of intermediate cell density the nuclei appeared almost touching and in high density tumours they appeared to be overlapping in these sections.) Other features that were recorded, but not incorporated into the grading system, were calcification and the proportion showing clear cell change, that is, the classical "frog spawn" appearance of oligodendroglioma. The grading system was based on sections stained with haematoxylin and eosin. In addition all tumours were studied with the immunoperoxidase, PAP method, after Sternberger, to demonstrate GFAP. ${ }^{10}$ Antibodies used were polyclonal anti-GFAP (Dako) and swineanti-rabbit IgG (Dako). Negative control sections were included.

\section{Results}

\section{Clinical features}

The age and sex characteristics of the 42 patients are given in table 2 . The age at presentation ranged from 7 to 69 years, with a mean of 44 years. The sex incidence was almost equal. The duration of the clinical history and the nature of the presenting symptom are

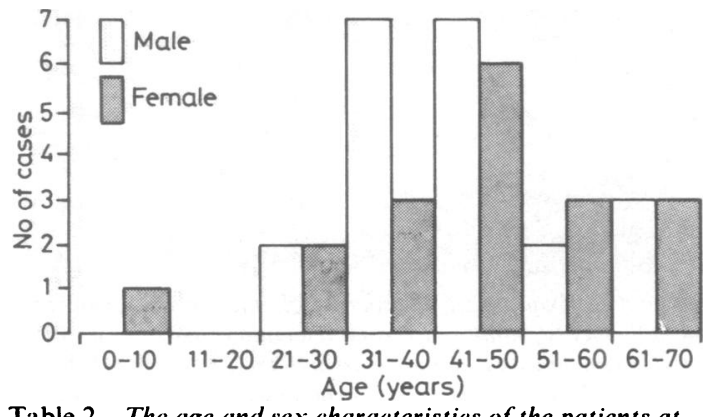

Table 2 The age and sex characteristics of the patients at the time of diagnosis. shown in tables 3 and 4 . Twenty-two patients gave a history longer than one year. Of the 19 patients whose first symptom was epilepsy, 15 gave a history longer than one year, nine more than 5 years. Of the 23 patients whose first symptom was something other than epilepsy, such as raised intracranial pressure or focal neurological deficit, 16 gave a history of less than one year's duration. The first symptom was grand mal epilepsy in 13 patients, focal epilepsy in six patients, clinical evidence of raised intracranial pressure in 13 patients, the gradual evolution of a focal neurological deficit in seven patients and sudden onset of focal neurological deficit (stroke-like) in

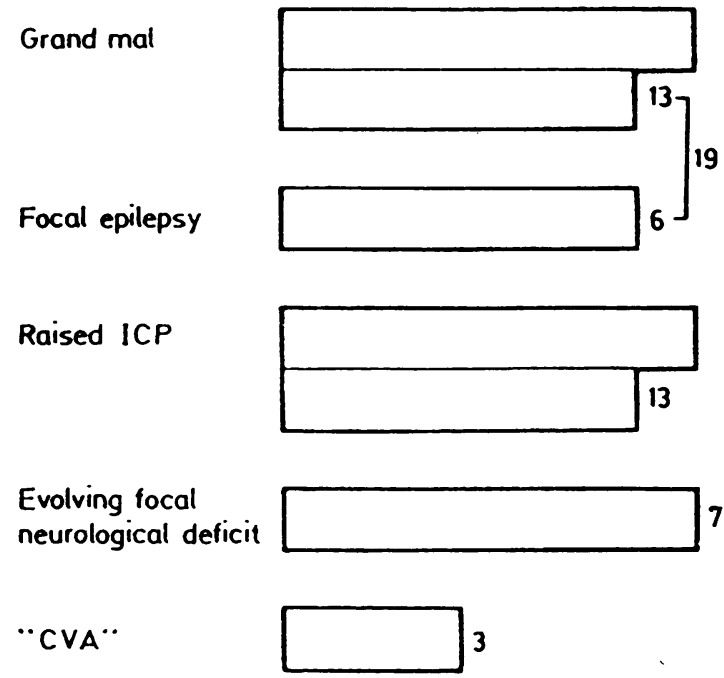

Table 3 The number of patients showing each of the five presenting symptoms.

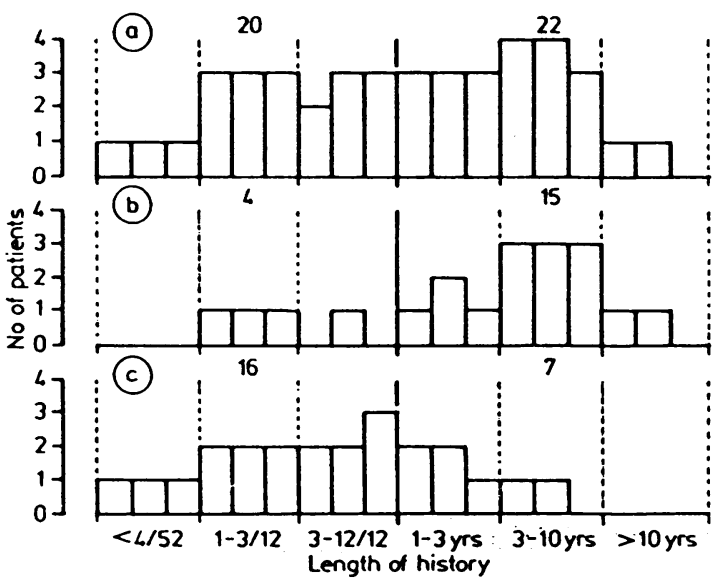

Table 4 The duration of the clinical history in the whole group of patients; $(a)$ in patients whose presenting symptom was epilepsy; and $(b)$ in patients whose presenting symptom w'as something other than epilepsy $(c)$. 
three. In the stroke-like presentations the clinical picture was of recurrent transient ischaemic attacks in one instance, of cerebral infarction in another and of fatal intracerebral haemorrhage in the third. Although table 3 illustrates the first symptom of each patient, it is important to note that by the time of diagnosis 24 of the 42 patients had suffered epileptic attacks, 25 had clinical evidence of raised intracranial pressure and 25 had localising neurological signs appropriate to the site of the lesion.

Figure 1 shows the anatomical location of the tumours. The frontal predominance is noteworthy. Twenty-two tumours involved the right cerebral hemisphere, 16 the left and four were midline, involving both cerebral hemispheres. There were no infratentorial tumours in this series. Figure 2 endeavours to show the relationship between the nature of the first symptom and tumour site. However, the nature of the first symptom did not show good correlation with the intracerebral location with the possible exception of tumours situated in the fronto-temporal or temporal region, of which seven

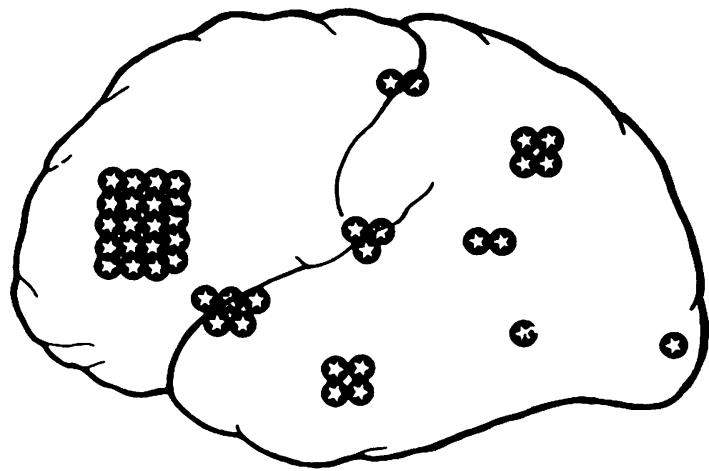

Fig 1 A diagram to show the location of the 42 tumours.

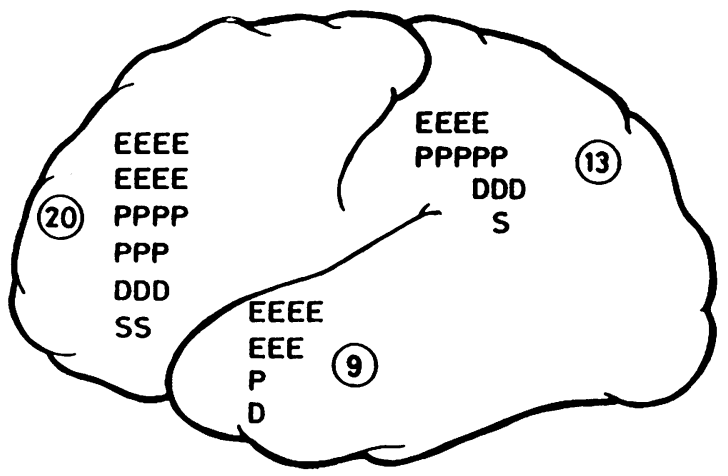

$$
E=\text { Epilepsy } P=41 C P \quad S=C V A \quad D=\begin{gathered}
\text { Progressive focal } \\
\text { neuro. deficit }
\end{gathered}
$$

Fig 2 A diagram to show the relationship between presenting symptom and tumour location. out of nine presented with epilepsy as their first symptom.

Calcification of the tumour was diagnosed preoperatively in $21(50 \%)$ patients, in 18 of 36 who had skull radiographs and in 12 of 24 who had a CT scan, (some patients had both investigations).

The surgical treatment was biopsy only in 10 cases, subtotal removal in 18 and macroscopic removal in 14 patients. Thirty-eight patients had a full course of radiotherapy immediately post-operatively, consisting of between 4,000 and 4,950 rad administered in 15 to 20 fractions. Three patients declined very rapidly after their surgical treatment and were never able to undergo a full course of radiotherapy. One patient did not receive radiotherapy after the initial surgery, but it was given with benefit 18 months later for tumour recurrence. Thus in this series there is only one patient who has survived for several years who did not receive early radiotherapy. The majority of patients in this survey received practically the same management, a major surgical resection followed by radiotherapy.

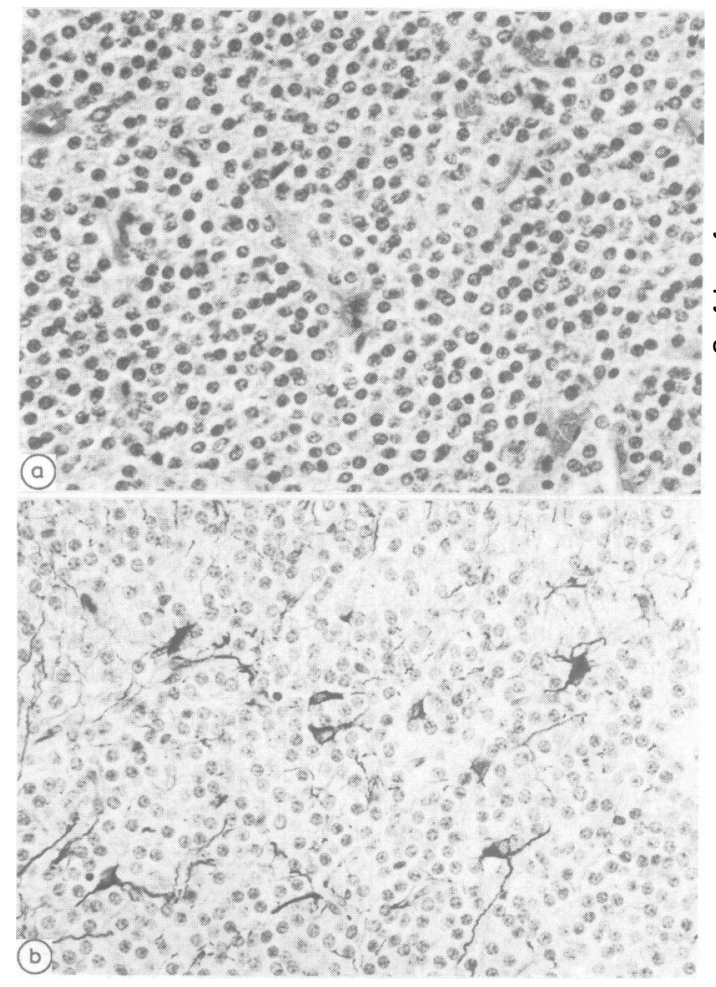

Fig 3 (a) Example of oligodendroglioma Grade 1, showing low cell density, small, uniform nuclei and absence of mitotic activity (haematoxylin and eosin $\times 50$ ) (b) Large GFAP positive cells, with astrocytic morphology, amongst smaller negative tumour cells. (Same tumour as (a)) (immunoperoxidase method for GFAP $\times 50$ ). 
At the time of data collection in July 1984 the outcome of treatment was known in 41 of the 42 patients. The one remaining patient was lost to follow-up 4 years after operation. The median survival time was 44 months and only 14 patients were known to be alive in July 1984. Ten patients had survived for 5 years or more, the longest survivor remaining alive and well 17 years after treatment. Nine of the patients had died within a year of treatment, one as a result of pulmonary embolism, but as a direct result of the tumour in the other eight.

\section{Histological findings}

Re-assessment of the original haematoxylin and eosin stained sections in 1984 confirmed the original diagnosis of oligodendroglioma. In two cases only, the possibility of an astrocytomatous component was queried at this stage, but because of a definite preponderance of distinctly oligodendrogliomatous tissue these cases were retained. Histological grading, (as set out in table 1 and illustrated in fig $3 a, 4 a$ and $5 a$ )

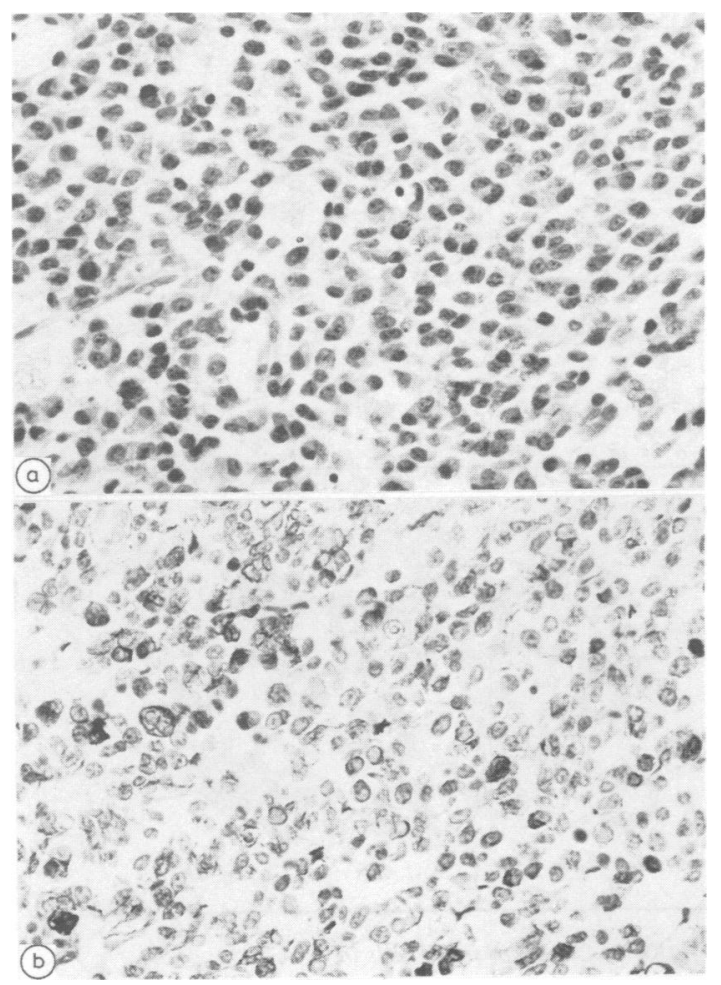

Fig 4 (a) Example of oligodendroglioma Grade 2, showing moderate nuclear pleomorphism and occasional mitotic figures. (haematoxylin and eosin $\times 50$ ) (b) Scattered small tumour cells show' positive reaction for GFAP

(immunoperoxidase method for GFAP $\times 50$ ) (same tumour as (a)). assigned 11 tumours to grade 1 , seven to grade 2 and the majority, 24 tumours to grade 3 . The biopsies were frequently non-uniform (14/42 cases). Foci of low cell density with regular rounded small nuclei coexisted with areas showing obvious nuclear pleomorphism. Large areas of clear cell change correlated best with low grade tumours, although two grade 1 tumours did not contain any such foci. These were examples of oligodendroglioma formed of cells with abundant eosinphilic cytoplasm and slightly eccentric nuclei, a well recognised appearance.

Calcification was detected in 15 tumours, in the form of calcospherities of variable density. Occasionally the calcification appeared greatest in the non tumourous tissue at the edge of the biopsy. Histological evidence of calcification did not correlate very closely with the radiological findings. Although in 25/42 cases there was agreement on either positive or negative findings, there was a discrepancy in 15 cases (radiological data were not available in two cases). In eight of these 15 cases calcification was only detected

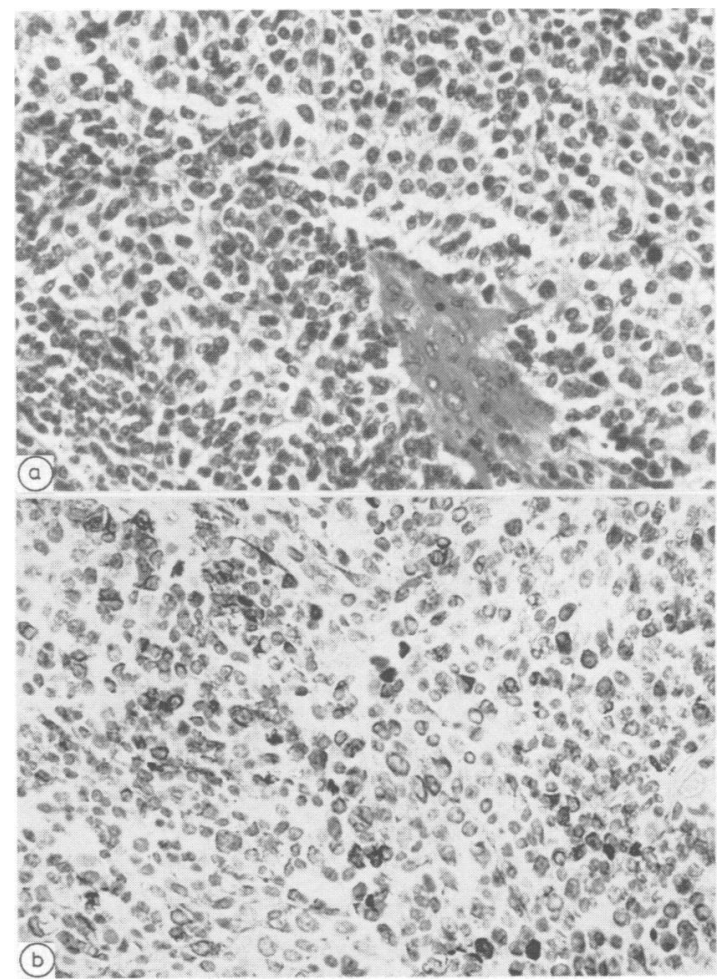

Fig 5 (a) Example of oligodendroglioma Grade 3, showing high cell density, severe nuclear pleomorphism and frequent mitoses. (Haematoxylin and eosin $\times$ 50) (b) Many of the tumour cells are GFAP positive. There is variation in cell size and shape, but the larger cells do not have typical morphology of fibrous astrocytes. (immunoperoxidase method for GFAP $\times 50$ ) (same tumour as (a)). 
by radiology and in the other seven only by histology.

Cells staining positively for GFAP were detected in almost every tumour (figs $3 \mathrm{~b}, 4 \mathrm{~b}$ and $5 \mathrm{~b}$ ). (Reserve paraffin embedded tissue was not available in one case). Several distinctive patterns emerged. Large biopsies with non-uniform histology frequently exhibited more than one pattern. The staining reactions are illustrated diagrammatically in fig 6 .

In patterns 1 and 2 the large cells with long pro- cesses were interpreted as reactive astrocytes, because they appeared to be a completely different population from the small rounded tumour cells with no transitional forms and also because identical patterns can be seen in non-glial neoplasms, such as malignant cerebral lymphoma. The astrocytic network pattern was particularly characteristic of the grade 1 tumour, present in all but $1 / 11$ tumours. However the finding of reactive astrocytes within the tumour was not

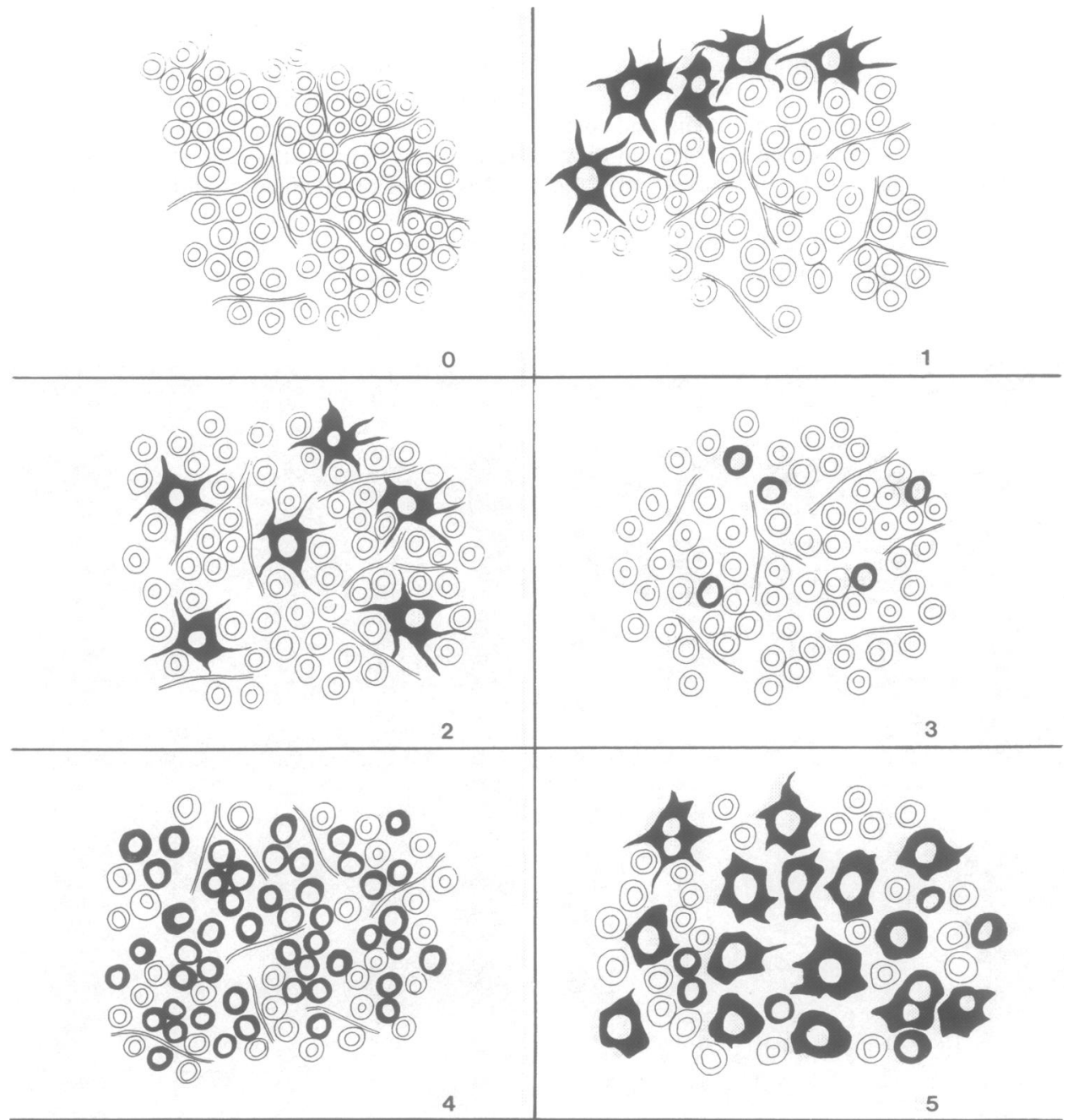

Fig 6 Diagrams to summarise the appearance and distribution of GFAP positive cells in oligodendrogliomas. Positive cells are indicated by dark cytoplasm:

(0) Large areas of tumour are completely negative

(1) Positive cells are present at the periphery of the tumour and are larger than the majority of unstained tumour cells. The positive cells have long cytoplasmic processes suggesting they are fibrous astrocytes.

(2) Positive cells, resembling fibrous astrocytes, are dispersed in a network pattern amongst smaller unstained tumour cells, as shown in fig $3 b$.

(3) Positive cells are few in number, but are the same size and shape as unstained small tumour cells.

(4) Positive cells have the same morphology as pattern 3, but the majority of tumour cells are stained, as shown in figs $4 b$ and $5 b$.

(5) Large astrocyte-like and smaller tumour cells are both positive with transitional forms. 
confined to low grade neoplasm and was also present in several of the more pleomorphic high grade tumours. In patterns 3 and 4 the positive cells were interpretated as neoplastic cells because they were morphologically indistinguishable from unstained tumour cells. This cell type has been recognised by other investigators and labelled glial fibrillary oligodendrocyte, (GFOC), by one. ${ }^{11}$ This staining pattern was seen in one grade 1 tumour and in several intermediate and high grade tumours (figures $4 b$ and $5 b$ ).

\section{Factors predicting outcome}

Factors which might have predictive value were assessed by the use of survival curves, calculated by the life table method, which were compared by using the log rank test. ${ }^{1213}$ Statistically significant correlations have been achieved in this group of patients by this method. In fig 7 the survival curve of patients aged 45 years and over is compared with that of patients less than 45 years, (the mean age for the whole group at the time of diagnosis was 44 years). The better survival of the younger group compared with the older patients is highly significant $(\mathrm{p}=$ 0.0001 ).

Of the other clinical features at the time of diagnosis, the only factor to achieve statistical significance

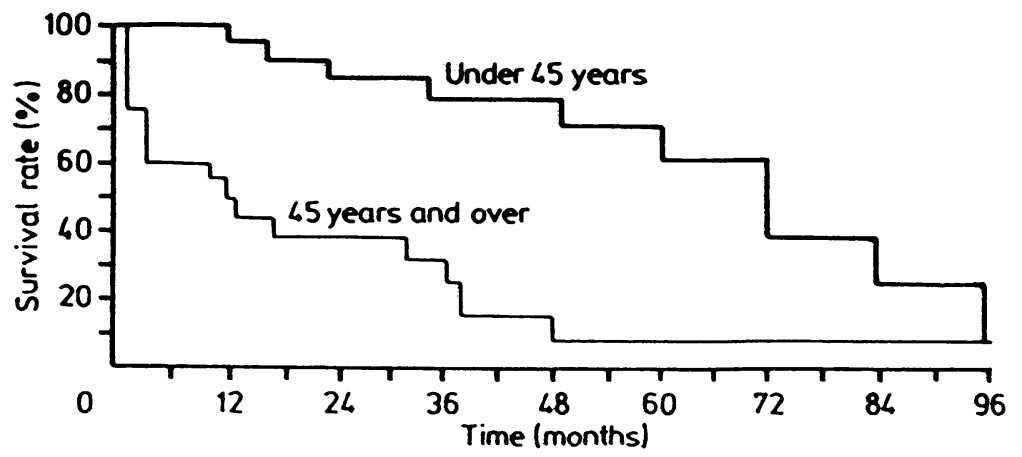

Fig 7 Survival curves to show the predictive value of age at the time of presentation.

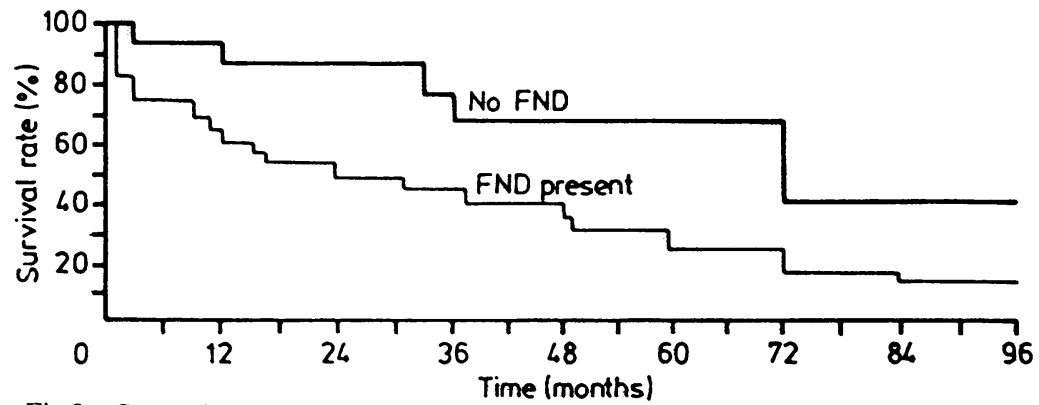

Fig 8 Survival curves to show the predictive value of the presence of a focal neurological deficit at the time of presentation.

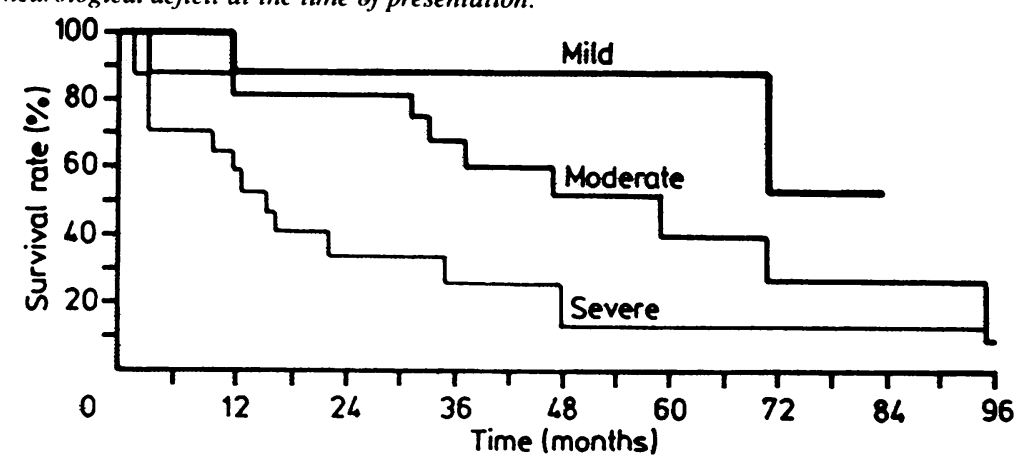

Fig 9 Survival curves to show the predictive value of the presence of nuclear pleomorphism in the tumour. 
was the presence of focal neurological deficit at the time of presentation. Figure 8 shows the difference in survival between those with, and those without such a deficit. This difference is statistically significant, $(p=$ 0.0242). Of the histological features of the tissue removed at the time of diagnosis, nuclear pleomorphism was the single characteristic to show a statistically significant correlation with survival, $(p=$ 0.0127 ), as shown in fig 9 .

Other factors which showed trends by this method of analysis, but which did not achieve statistical significance, include the duration of symptoms prior to diagnosis, radiological evidence of calcification, mitotic activity in the tumour and its overall histological grade. Patients with a long history, with a calcified tumour, with low mitotic rates and low histogical grade fared better, but the trends in the survival curves did not achieve statistical significance for these factors.

Trends for other clinical, treatment and histological characteristics were sought but not found. Hence, there was no demonstrable correlation between survival time and the following clinical features; sex, nature of the first symptom, occurrence of epilepsy, raised intracranial pressure or location of the tumour. The different surgical procedures did not produce any significant differences in survival time and there was equally negative correlation with the following histological features; necrosis, vascular endothelial cell proliferation, clear cell change and the various patterns of GFAP containing cells in the tumour.

\section{Discussion}

Analysis of the comprehensive data collected from these patients has clarified the common clinical features of oligodendrogliomas and has revealed certain clinical and pathological factors of prognostic significance. Following a brief preliminary communication, ${ }^{14}$ the results are now discussed in detail.

In the first category this series has emphasised that oligodendrogliomas are tumours of the cerebral hemispheres in adults. The fact that over 20 years in a regional neurosurgical centre only one oligodendroglioma has been diagnosed in childhood has confirmed their rarity in this age group. Likewise, subtentorial oligodendrogliomas are unusual and there were none in this group. A frequent frontal location has been confirmed. A long preceding history of epilepsy and radiological tumour calcification are also common. It must be emphasised however that half the patients in this study gave a history of less than a year, half the patients did not present with epilepsy as their initial symptom, and half the patients showed no calcification of their tumour on skull radiographs or CT scan. Clearly diagnostic thoughts of oligodendro- glioma cannot be confined to patients with a calcified lesion presenting with a long history of epilepsy.

In the second category, the first observation must be the rather poor overall prognosis for patients with oligodendroglioma. Whilst comparing well with the prognosis for patients with grade 3 and 4 astrocytomas, ${ }^{15}$ the outlook for patients with oligodendroglioma in this series was only fair, $(9 / 42$ patients, $21 \%$, dying within 1 year and only $10 / 42$ patients, $24 \%$, surviving 5 years from the time of diagnosis and treatment). This outcome is similar to several reports on survival in the literature, but not as satisfactory as the figures produced by Smith et al, $48 \% 5$ year survival $^{16}$ and by Chin et al, $60 \% 5$ year survival. ${ }^{3}$

Previous workers have had difficulty in establishing any clinical factors which have correlated reliably with prognosis. The unpredictability of oligodendroglioma is a feature of many reports. In this study, age at the time of diagnosis has been shown to be an overriding and statistically significant factor. In this fairly small series it is not possible to determine whether the adverse effect of age is solely due to the inability of older patients to withstand the presence of a brain tumour and the effects of anaesthesia and major surgery, or whether there is a genuine difference in the biological behaviour of the tumours. Even if it is not the only factor, it seems likely that comparatively poorer general health must to some extent be responsible for the more rapid demise of the older patients. If so, even more rigorous attention to pre-operative and post-operative care may increase the survival in this group. In this series, eight patients from the older group died within the first few postoperative weeks, (one from pulmonary embolus, but? the rest from the direct effects of their brain tumour). All of the younger patients survived the postoperative period and the earliest death in this group was at 1 year. The series is too small to prove a significant biological difference in the tumours of older patients, but it is of interest that the majority of grade 1 tumours, eight out of 11 , were identified in the younger patients.

The statistically significant correlation of poor prognosis with the presence of a focal neurological deficit at the time of diagnosis has not been reported previously and, along with age, becomes one of the two useful clinical factors to remember when counselling patients with oligodendriglioma and their relatives.

The value of histological grading has long been a source of controversy in oligodendroglioma. Smith et al have advocated grading and have shown a correlation between histological features and survival, ${ }^{16}$ whereas others have claimed histology was irrelevant. ${ }^{12}$ In this series there is a definite trend shown in the survival curve for grade 1 and grade 2 tumours to 
fare better than grade 3. This did not achieve statistical significance but this is probably because the numbers in each group were too small. However, as in a previous survey, nuclear pleomorphism alone has proved to be a significant prognostic factor. The tumours were graded as in the Kernohan system for astrocytomas with the additional refinement of a point scoring system, and grade 3 and 4 tumours were included in a single category. The majority of neuropathologists employ this system for astrocytomas and at present we would advocate its application to oligodendrogliomas, whilst accepting that as more oligodendrogliomas are assessed, different weighting may be needed for individual morphologic criteria.

Immunoperoxidase staining for GFAP was performed in the hope of identifying new histological prognostic factors. This hope was not fulfilled. Cells interpreted as reactive astrocytes were found in both low grade and high grade tumours and their presence showed no correlation with survival. However, this method may yet prove useful to distinguish different types of oligodendroglioma and to segregate mixed tumours, that is tumours which are partly oligodendrogliomatous, but also contain neoplastic astrocytes. Tumours in which the two malignant components are quite separate are generally recognisable with haematoxylin and eosin. Tumours of this type were excluded from this series. However there does appear to be a category, particularly well demonstrated by GFAP, with a spectrum of positive cells from small round oligodendrocyte like cells to large pleomorphic cells with astrocytic morphology. There were only two tumours in this category, both classed as grade 3. It may be anticipated that these will behave as malignant astrocytomas, but more data are required. Until a reliable and specific immunological marker for oligodendrocytes is found, the inclusion of GFAP staining in the histological assessment of oligodendrogliomas will help to eliminate mixed tumours from any survey and thus assist recognition of comparable tumours for inclusion in therapeutic trials.

The identification of GFAP in well differentiated oligodendrogliomas is at first surprising as it is generally regarded as a marker of astrocytic differentiation. However, a recent study of developing myelin suggests that GFAP is expressed transiently in developing oligodendrocytes. ${ }^{17}$ If this is confirmed, in this situation GFAP can be regarded as another oncofetal antigen.

Despite the large volume of data collected from a major neurosurgical centre the number of patients is too small for significant results to be obtained from a multivariate analysis; nevertheless certain trends have emerged which indicate the need for further detailed documentation of oligodendroglioma. On present evidence it seems that the general optimism which greets diagnosis of oligodendroglioma, as opposed to malignant astrocytoma, is justified, provided the patient is a young adult. In this series young age outweighed histological evidence of malignancy. The prognosis of all grades of oligodendroglioma in young adults is far better than that for grade 3 and 4 astrocytoma. Likewise benign histology in an older patient does not guarantee a favourable outcome and in the older group the prognosis, whilst not as poor, approaches that of high grade astrocytoma.

We thank, most sincerely, Dr Lawrence Freedman for his very considerable help in the statistical analyses presented in this paper.

\section{References}

1 Russell DS, Rubenstein LJ. Oligodendroglioma. In: Pathology of Tumours of the Nervous System. London. Edward Arnold Ltd 1977:195-203.

2 Roberts M, German WJ. A long term study of patients with oligodendrogliomas. $J$ Neurosurg 1966;24: 697-700.

3 Chin HW, Hazel JJ, Kim TH, Webster JH. Oligodendrogliomas. Cancer 1980;45:1458-66.

4 Reedy DP, Bay JW, Hahn JF. Role of irradiation therapy in the treatment of cerebral oligodendroglioma: an analysis of 57 cases and a literature review. Neurosurgery 1983;13:499-503.

5 Kernohan JW, Sayre GP. Tumours of the central nervous system. In: Atlas of Tumour Pathology, section 10 fascicles 35 and 37. Washington DC, Armed Forces Institute of Pathology, 1952.

6 Burger PC, Vollmer RT. Histologic features of prognostic significance in the glioblastoma multiforme. Cancer 1980;46:1179-86.

7 Nelson JS, Scheonfeld D, Tsukada Y, Fulling K. Histologic criteria with prognostic significance for malignant glioma. In: Chang $\mathrm{CH}$, Housepian EM, eds. Tumours of the Central Nervous System. New York: Masson Publishing, 1982:1-4.

8 Byar DP, Green SB, Strike TA. Prognostic factors for malignant glioma. In: Walker MD, ed. Oncology of the Nervous System. The Hague. Martinus Nịhoff 1983:397-5.

9 Chang CH, Horton J, Schenfeld D, et al. Comparison of postoperative and combined postoperative radiotherapy and chemotherapy in the multidisciplinary management of malignant gliomas. Cancer 1983;52:997-1007.

10 Sternberger LA. The unlabelled antibody peroxidaseantiperoxidase (PAP) method. In: Immunocytochemistry. New York: John Wiley 1979:104-70.

11 Herpers MJHM, Budka H. Glial fibrillary acidic protein (GFAP) in oligodendroglial tumours: gliofibrillary oligodendroglioma and transitional oligoastrocytoma as subtypes of oligodendroglioma. Acta Neuropathol (Berl) 1984;64:265-72. 
12 Kaplan EL, Meier PJ. Non-parametric estimation from incomplete observations. J Am Statistical Association 1958;53:457-81.

13 Peto R, Pike MC, Armitage P. Design and analysis of randomised trials requiring prolonged observation of each patient. 2. Analysis and examples. $\mathrm{Br} J$ Cancer 1977;35:1-39.

14 Anderson JR, Wilkinson IMS, Holmes AE. Oligodendroglioma - a clinicopathological study of 40 cases diagnosed in a 20 year period. Neuropathol Appl Neurobiol 1985;11:505-6.
15 MRC. Working Party on Misonidazole in Gliomas. A study of the effect of Misonidazole in conjunction with radiotherapy in the treatment of grades 3 and 4 astrocytomas. Br J Radiol 1983;56:673-82.

16 Smith MT, Ludwig CL, Godfrey AD, Armbrustmacher VW. Grading of oligodendrogliomas. Cancer 1983;52:2107-14.

17 Choi BH, Kim RC. Expression of glial fibrillary acidic protein in immature oligodendroglia. Science 1984;223:407-9. 